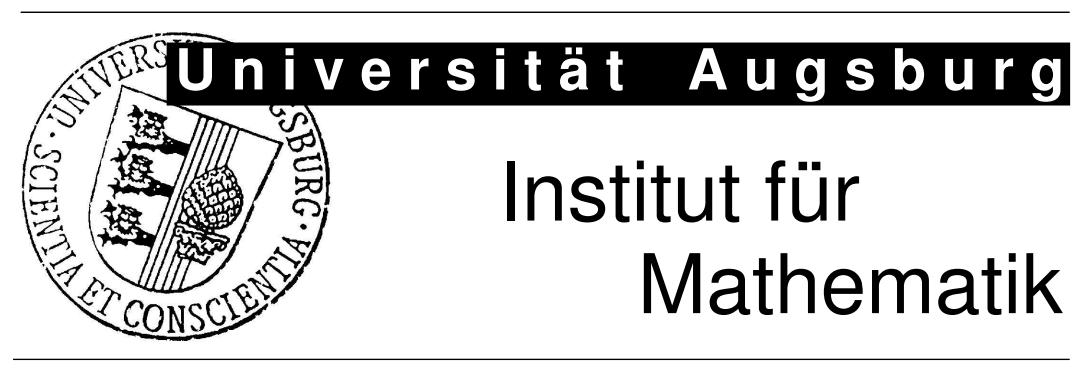

Ronald H.W. Hoppe, Natasha S. Sharma

Convergence Analysis of an Adaptive Interior Penalty Discontinuous Galerkin Method for the Helmholtz Equation 


\section{Impressum:}

\section{Herausgeber:}

Institut für Mathematik

Universität Augsburg

86135 Augsburg

http://www.math.uni-augsburg.de/pages/de/forschung/preprints.shtml

\section{ViSdP:}

Ronald H.W. Hoppe

Institut für Mathematik

Universität Augsburg

86135 Augsburg

Preprint: Sämtliche Rechte verbleiben den Autoren (C) 2011 


\title{
CONVERGENCE ANALYSIS OF AN ADAPTIVE INTERIOR PENALTY DISCONTINUOUS GALERKIN METHOD FOR THE HELMHOLTZ EQUATION
}

\author{
R. H. W. HOPPE*† AND N. SHARMA*
}

\begin{abstract}
We are concerned with a convergence analysis of an adaptive Interior Penalty Discontinuous Galerkin (IPDG) method for the numerical solution of acoustic wave propagation problems as described by the Helmholtz equation. The mesh adaptivity relies on a residual-type a posteriori error estimator that does not only control the approximation error but also the consistency error caused by the nonconformity of the approach. As in the case of IPDG for standard second order elliptic boundary value problems, the convergence analysis is based on the reliability of the estimator, an estimator reduction property, and a quasi-orthogonality result. However, in contrast to the standard case, special attention has to be paid to a proper treatment of the lower order term in the equation containing the wavenumber which is taken care of by an Aubin-Nitsche type argument for the associated conforming finite element approximation. Numerical results are given for an interior Dirichlet problem and a screen problem illustrating the performance of the adaptive IPDG method.
\end{abstract}

Key words. Interior Penalty Discontinuous Galerkin method, Helmholtz equation, adaptivity, convergence analysis

AMS subject classifications. 65N30, 65N50, 78A45, 78M10

1. Introduction. Let $\Omega_{D}$ and $\Omega_{R}$ be bounded polygonal domains in $\mathbb{R}^{2}$ such that $\Omega_{D} \subset \Omega_{R}$. We set $\Omega:=\Omega_{R} \backslash \Omega_{D}$ and note that $\partial \Omega=\Gamma_{D} \cup \Gamma_{R}$ where $\Gamma_{D}:=\partial \Omega_{D}$ and $\Gamma_{R}:=\partial \Omega_{R}$. Given complex valued functions $f$ in $\Omega$ and $g$ on $\Gamma_{R}$, we consider the Helmholtz problem

$$
\begin{aligned}
-\Delta u-k^{2} u=f & & \text { in } \Omega, \\
\frac{\partial u}{\partial \nu_{R}}+i k u=g & & \text { on } \Gamma_{R}, \\
u=0 & & \text { on } \Gamma_{D},
\end{aligned}
$$

which describes an acoustic wave with wavenumber $k>0$ scattered at the sound-soft scatterer $\Omega_{D}$. In (1.1b), $\nu_{R}$ denotes the exterior unit normal at $\Gamma_{R}$ and $i$ stands for the imaginary unit.

Finite element methods for acoustic wave propagation problems such as (1.1a)-(1.1c) have been widely studied in the literature (cf., e.g., $[4,12,15,28,30]$ as well as the survey article [17], the monographs [27, 29] and the references therein). In case of large wavenumbers $k$, the finite element discretization typically requires fine meshes for a proper resolution of the waves and thus results in large linear algebraic systems to be solved. Moreover, the use of standard adaptive mesh refinement techniques based on a posteriori error estimators is marred by the pollution effect $[5,27]$. Recently, Discontinuous Galerkin (DG) methods [14, 24, 34] have been increasingly applied to wave propagation problems in general [13] and the Helmholtz equation in particular $[2,3,18,19,20,21]$ including hybridized DG approximations [23]. An a posteriori error analysis of DG methods for standard second order elliptic boundary value problems has been performed in $[1,8,10,26,31,35]$, and a convergence analysis has been

\footnotetext{
*Dept. of Math., Univ. of Houston, Houston, TX 77204-3008, U.S.A.

†Inst. of Math., Univ. of Augsburg, D-86159 Augsburg, Germany

$\ddagger$ The authors acknowledge support by NSF grant DMS-0810176
} 
provided in $[9,25,32]$. However, to the best of our knowledge a convergence analysis for adaptive DG discretizations of the Helmholtz equation is not yet available in the literature.

It is the purpose of this paper to provide such a convergence analysis for an Interior Penalty Discontinuous Galerkin (IPDG) discretization of (1.1a)-(1.1c) based on a residual type a posteriori error estimator featuring element and edge residuals. The paper is organized as follows:

In section 2, we introduce the adaptive IPDG method, discuss the consistency error due to the nonconformity of the approach, and present the residual a posteriori error estimator as well as the marking strategy (Dörfler marking) for adaptive mesh refinement. Section 3 shows that the consistency error can be controlled by the estimator, provides an estimator reduction property in the spirit of [11] and establishes the reliability of the estimator. Another important ingredient of the convergence analysis is a quasi-orthogonality result that will be dealt with in section 4 . The particular difficulty we are facing here is the proper treatment of the lower order term in (1.1a)containing the wavenumber $k$. Adopting an idea from from the convergence analysis of adaptive conforming edge element approximations of the time-harmonic Maxwell equation [39], we use the conforming approximation of (1.1a)-(1.1c) and take advantage of an Aubin-Nitsche type argument (cf. Lemma (4.4)). Hence, the quasi-orthogonality of the IPDG approximation can be established by invoking the associated conforming approximations (cf. Theorem 4.1). Combining the reiliability of the estimator, the estimator reduction property, and the quasi-orthogonality result, in section 5 we prove convergence of the adaptive IPDG in terms of a contraction property for a weighted sum of the discretization error in the mesh dependent energy norm and the error estimator. Finally, section 6 is devoted to a documentation of numerical results that illustrate the performance of the adaptive IPDG over a wide range of wavenumbers.

2. The adaptive IPDG method. The functions considered in this paper are complex-valued. For a complex number $z \in \mathbb{C}$ we denote by $\operatorname{Re}(z), \operatorname{Im}(z)$ its real and imaginary part such that $z=\operatorname{Re}(z)+i \operatorname{Im}(z), \bar{z}:=\operatorname{Re}(z)-i \operatorname{Im}(z)$ is the complex conjugate of $z$ and $|z|:=\sqrt{\operatorname{Re}(z)^{2}+\operatorname{Im}(z)^{2}}$ stands for the absolute value. We further adopt standard notation from Lebesgue and Sobolev space theory (cf., e.g., [38]). In particular, for $D \subseteq \Omega$ we refer to $L^{2}(D)$ and $H^{s}(D)$ as the Hilbert space of Lebesgue integrable complex-valued functions in $D$ with inner product $(\cdot, \cdot)_{0, D}$ and associated norm $\|\cdot\|_{0, D}$ and the Sobolev space of complex-valued functions with inner product $(\cdot, \cdot)_{s, D}$ and norm $\|\cdot\|_{s, D}$. For $\Sigma \subseteq \partial D$ and a function $v \in H^{s}(D)$, we denote by $\left.v\right|_{\Sigma}$ the trace of $v$ on $\Sigma$.

Under the following assumption on the data of the problem

$$
f \in L^{2}(\Omega), \quad g \in L^{2}\left(\Gamma_{R}\right)
$$

the weak formulation of (1.1a)-(1.1c) amounts to the computation of $u \in V, V:=$ $H_{0, \Gamma_{D}}^{1}(\Omega):=\left\{v \in H^{1}(\Omega)|v|_{\Gamma_{D}}=0\right\}$ such that for all $v \in V$ it holds

$$
a(u, v)-k^{2} c(u, v)+i k r(u, v)=\ell(v) .
$$


Here, the sesquilinear forms $a, c, r$ and the linear functional $\ell$ are given by

$$
\begin{aligned}
& a(u, v):=\int_{\Omega} \nabla u \cdot \nabla \bar{v} d x, \quad c(u, v):=\int_{\Omega} u \bar{v} d x, \\
& r(u, v):=\int_{\Gamma_{R}} u \bar{v} d s, \quad \ell(v):=\int_{\Omega} f \bar{v} d x+\int_{\Gamma_{R}} g \bar{v} d s .
\end{aligned}
$$

REMARK 2.1. It is well-known that (2.2) satisfies a Fredholm alternative (cf., e.g., [33]). In particular, if $k^{2}$ is not an eigenvalue of $-\Delta$ subject to the boundary conditions (1.1b),(1.1c), for any $f, g$ satisfying (2.1) there exists a unique solution $u \in V$. In this case, the sesquilinear form $\hat{a}(\cdot, \cdot):=a(\cdot, \cdot)-k^{2} c(\cdot, \cdot)+i k r(\cdot, \cdot)$ satisfies the inf-sup conditions

$$
\inf _{v \in V} \sup _{w \in V} \frac{|\hat{a}(v, w)|}{\|v\|_{1, \Omega}\|w\|_{1, \Omega}}=\inf _{w \in V} \sup _{v \in V} \frac{|\hat{a}(v, w)|}{\|v\|_{1, \Omega}\|w\|_{1, \Omega}}>\beta
$$

hold true with a positive constant $\beta$ depending only on $\Omega$ and on the wavenumber $k$.

For the formulation of the IPDG method, we assume $\mathcal{H}$ to be a null sequence of positive real numbers and $\left(\mathcal{T}_{h}(\Omega)\right)_{h \in \mathcal{H}}$ a shape-regular family of simplicial triangulations of $\Omega$. For an element $T \in \mathcal{T}_{h}(\Omega)$, we denote by $h_{T}$ the diameter of $T$ and set $h:=$ $\max \left\{h_{T} \mid T \in \mathcal{T}_{h}(\Omega)\right\}$. For $D \subset \bar{\Omega}$, we refer to $\mathcal{E}_{h}(D)$ as the set of edges of $T \in \mathcal{T}_{h}(\Omega)$ in $D$. For $E \in \mathcal{E}_{h}(D)$, we denote by $h_{E}$ the length of $E$ and to $\omega_{E}:=\bigcup\{T \in$ $\left.\mathcal{T}_{H}(\Omega) \mid E \subset \partial T\right\}$ as the patch consisting of the union of elements sharing $E$ as a common edge. Moreover, $\mathcal{P}_{N}(D), N \in \mathbb{N}$, stands for the set of complex-valued polynomials of degree $\leq N$ on $D$. In the sequel, for two mesh dependent quantities $A$ and $B$ we use the notation $A \lesssim B$, if there exists a constant $C>0$ independent of $h$ such that $A \leq C B$.

We introduce the finite element spaces

$$
\begin{array}{r}
V_{h}:=\left\{v_{h}: \bar{\Omega} \rightarrow \mathbb{C}\left|v_{h}\right|_{T} \in \mathcal{P}_{N}(T), T \in \mathcal{T}_{H}(\Omega)\right\}, \\
\mathbf{V}_{h}:=\left\{\mathbf{v}_{h}: \bar{\Omega} \rightarrow \mathbb{C}^{2}\left|\mathbf{v}_{h}\right|_{T} \in \mathcal{P}_{N}(T)^{2}, T \in \mathcal{T}_{H}(\Omega)\right\} .
\end{array}
$$

Functions $v_{h} \in V_{h}$ are not continuous across interior edges $E \in \mathcal{E}_{H}(\Omega)$. For $E:=$ $T_{+} \cap T_{-}, T_{ \pm} \in \mathcal{T}_{H}(\Omega)$, we denote by $\left\{v_{h}\right\}_{E}$ the average of $v_{h}$ on $E$ and by $\left[v_{h}\right]_{E}$ the jump of $v_{h}$ across $E$ according to

$$
\left\{v_{h}\right\}_{E}:=\frac{1}{2}\left(\left.v_{h}\right|_{E \cap T_{+}}+\left.v_{h}\right|_{E \cap T_{-}}\right), \quad\left[v_{h}\right]_{E}:=\left.v_{h}\right|_{E \cap T_{+}}-\left.v_{h}\right|_{E \cap T_{-}}, \quad E \in \mathcal{E}_{h}(\Omega),
$$

and we define $\left\{v_{h}\right\}_{E},\left[v_{h}\right]_{E}, E \in \mathcal{E}_{h}(\Gamma)$, accordingly.

We introduce a mesh dependent sesquilinear form $a_{h}^{I P}: V_{h} \times V_{h} \rightarrow \mathbb{C}$ by means of

$$
\begin{aligned}
a_{h}^{I P}\left(u_{h}, v_{h}\right):= & \sum_{T \in \mathcal{T}_{h}(\Omega)}\left(\nabla u_{h}, \nabla v_{h}\right)_{0, T}-\sum_{E \in \mathcal{E}_{h}\left(\Omega \cup \Gamma_{D}\right)}\left(\left\{\frac{\partial u_{h}}{\partial \nu_{E}}\right\}_{E},\left[v_{h}\right]_{E}\right)_{0, E} \\
& -\sum_{E \in \mathcal{E}_{h}\left(\Omega \cup \Gamma_{D}\right)}\left(\left[u_{h}\right]_{E},\left\{\frac{\partial v_{h}}{\partial \nu_{E}}\right\}_{E}\right)_{0, E}+\sum_{E \in \mathcal{E}_{h}\left(\Omega \cup \Gamma_{D}\right)} \frac{\alpha}{h_{E}}\left(\left[u_{h}\right]_{E},\left[v_{h}\right]_{E}\right)_{0, E},
\end{aligned}
$$

where $\alpha>0$ is a suitably chosen penalty parameter.

The IPDG method for the approximation of the solution of (1.1a)-(1.1c) requires the 
computation of $u_{h} \in V_{h}$ such that for all $v_{h} \in V_{h}$ it holds

$$
a_{h}^{I P}\left(u_{h}, v_{h}\right)-k^{2} c\left(u_{h}, v_{h}\right)+i k r\left(u_{h}, v_{h}\right)=\ell\left(v_{h}\right) .
$$

We further define $u_{h}^{c} \in V_{h}^{c}:=V_{h} \cap H_{0, \Gamma_{D}}^{1}(\Omega)$ as the conforming finite element approximation of (1.1a)-(1.1c) satisfying

$$
a\left(u_{h}^{c}, v_{h}^{c}\right)-k^{2} c\left(u_{h}^{c}, v_{h}^{c}\right)+i k r\left(u_{h}^{c}, v_{h}^{c}\right)=\ell\left(v_{h}^{c}\right), \quad v_{h}^{c} \in V_{h}^{c} .
$$

REMARK 2.2. If $k^{2}$ is not an eigenvalue of $-\Delta$ subject to the boundary conditions (1.1b),(1.1c), for sufficiently large penalty parameter $\alpha$ and sufficiently small mesh size $h$, the equations (2.6) and (2.7) have unique solutions $u_{h} \in V_{h}$ and $u_{h}^{c} \in V_{h}^{c}$ that continuously depend on the data. In particular, there exists $h^{*} \in \mathcal{H}, h^{*} \leq 1$, such that for $h \leq h^{*}$ the sesquilinear forms $\left.\hat{a}\right|_{V_{h}^{c} \times V_{h}^{c}}$ inherit (2.3), whereas the sesquilinear forms $\hat{a}_{h}^{I P}(\cdot, \cdot):=a_{h}^{I P}(\cdot, \cdot)-k^{2} c(\cdot, \cdot)+i k r(\cdot, \cdot)$ satisfy analogues of $(2.3)$ with positive inf-sup constants $\beta_{h}$ being uniformly bounded away from zero. Moreover, (2.6) is consistent with (2.2) in the sense that the solution $u \in V$ of (2.2) satisfies (2.6) for $v_{h}=v_{h}^{c} \in V_{h}^{c}$. In the sequel, we will always assume that $k^{2}$ is not an eigenvalue of $-\Delta$ and $h$ is sufficiently small such that (2.6) and (2.7) admit unique solutions.

We note that $a_{h}^{I P}(\cdot, \cdot)$ is not well defined on $V$. This can be remedied by means of a lifting operator $L: V+V_{h} \rightarrow \mathbf{V}_{h}$ according to

$$
\left(L(v), \mathbf{v}_{h}\right)_{0, \Omega}:=\sum_{E \in \mathcal{E}_{h}\left(\Omega \cup \Gamma_{D}\right)}\left([v]_{E}, \nu_{E} \cdot\left\{\mathbf{v}_{h}\right\}_{E}\right)_{0, E}, \quad v \in V+V_{h}, \mathbf{v}_{h} \in \mathbf{V}_{h} .
$$

As has been shown, e.g., in [37], the lifting operator is stable in the sense that there exists a constant $C_{L}>0$ depending only on the shape regularity of the triangulations such that

$$
\|L(v)\|_{0, \Omega}^{2} \leq C_{L} \sum_{E \in \mathcal{E}_{h}\left(\Omega \cup \Gamma_{D}\right)} h_{E}^{-1}\left\|[v]_{E}\right\|_{0, E}^{2}, \quad v \in V+V_{h} .
$$

On $V+V_{h}$, we define the mesh dependent DG norm

$$
\|v\|_{1, h, \Omega}:=\left(\sum_{T \in \mathcal{T}_{h}(\Omega)}\|\nabla v\|_{0, T}^{2}+\sum_{E \in \mathcal{E}_{h}\left(\Omega \cup \Gamma_{D}\right)} \alpha h_{E}^{-1}\left\|[v]_{E}\right\|_{0, E}^{2}\right)^{1 / 2},
$$

It is well known (cf., e.g., [9] and the references therein) that for sufficiently large penalty parameter $\alpha$ the DG-norm and the mesh dependent energy norm are equivalent, i.e., there exist constants $\alpha_{1}>0,0<\gamma<1$, and $C_{1}>0$ such that for all $\alpha \geq \alpha_{1}$ and $v \in V+V_{h}$ it holds

$$
a_{h}^{I P}(v, v) \geq \gamma\|v\|_{1, h, \Omega}^{2},
$$

whereas for all $\alpha \geq 1$ and $v, w \in V+V_{h}$ we have

$$
a_{h}^{I P}(v, w) \leq C_{1}\|v\|_{1, h, \Omega}\|w\|_{1, h, \Omega} .
$$

The DG approach is a nonconforming finite element method, since $V_{h}$ is not contained in $H_{0, \Gamma_{D}}^{1}(\Omega)$ due to the lack of continuity across interior edges $E \in \mathcal{E}_{h}(\Omega)$ and due to the enforcement of the homogeneous Dirichlet boundary condition (1.1c) by penalty 
terms on the edges $E \in \mathcal{E}_{h}\left(\Gamma_{D}\right)$. The nonconformity is measured by the consistency error

$$
\xi:=\inf _{v_{h}^{c} \in V_{h}^{c}}\left(\sum_{T \in \mathcal{T}_{h}(\Omega)}\left\|\nabla\left(u_{h}-v_{h}^{c}\right)\right\|_{0, T}^{2}\right)^{1 / 2} .
$$

We refer to $\Pi_{h}^{C}: V_{h} \rightarrow V_{h}^{c}$ as the Clément-type quasi-interpolation operator introduced in [9] such that for some constant $C_{A}>0$ depending only on the shape regularity of the triangulations it holds

$$
\begin{aligned}
& \sum_{|\beta|} \sum_{T \in \mathcal{T}_{h}(\Omega)}\left\|D^{\beta}\left(u_{h}-\Pi_{h}^{C} u_{h}\right)\right\|_{0, T}^{2} \leq \\
& C_{A} \sum_{E \in \mathcal{E}_{h}\left(\Omega \cup \Gamma_{D}\right)} h_{E}^{1-2|\beta|}\left\|\left[u_{h}\right]_{E}\right\|_{0, E}^{2}, \quad|\beta| \in\{0,1\} .
\end{aligned}
$$

It follows from (2.13) that

$$
\eta_{h, c}:=\left(\sum_{E \in \mathcal{E}_{h}\left(\Omega \cup \Gamma_{D}\right)} \eta_{E, c}^{2}\right)^{1 / 2}, \quad \eta_{E, c}:=h_{E}^{-1 / 2}\left\|\left[u_{h}\right]_{E}\right\|_{0, E} .
$$

Lemma 2.1. Let $u_{h} \in V_{h}$ and $u_{h}^{c} \in V_{h}^{c}$ be the solution of (2.6) and (2.7), respectively, and let $u_{h}^{n c}:=u_{h}-u_{h}^{c}$. Then, for $\alpha \geq 1$ there exists a positive constant $C_{n c}$, depending on $\beta, C_{1}$, and $C_{A}$, such that

$$
\sum_{T \in \mathcal{T}_{h}(\Omega)}\left\|u_{h}^{n c}\right\|_{1, T}^{2} \leq C_{n c} \alpha \eta_{h, c}^{2} .
$$

Proof. Obviously, we have

$$
\sum_{T \in \mathcal{T}_{h}(\Omega)}\left\|u_{h}^{n c}\right\|_{1, T}^{2} \leq 2 \sum_{T \in \mathcal{T}_{h}(\Omega)}\left(\left\|u_{h}-\Pi_{h}^{C} u_{h}\right\|_{1, T}^{2}+\left\|u_{h}^{c}-\Pi_{h}^{C} u_{h}\right\|_{1, T}^{2}\right) .
$$

It follows from (2.7) that $u_{h}^{c}-\Pi_{h}^{C} u_{h}$ satisfies

$$
\hat{a}\left(u_{h}^{c}-\Pi_{h}^{C} u_{h}, v_{h}^{c}\right)=\ell\left(v_{h}^{c}\right)-\hat{a}\left(\Pi_{h}^{C} u_{h}, v_{h}^{c}\right), \quad v_{h}^{c} \in V_{h}^{c} .
$$

Hence, in view of Remark 2.2 there exists a positive constant $C_{\beta}$ such that

$$
\left\|u_{h}^{c}-\Pi_{h}^{C} u_{h}\right\|_{1, \Omega} \leq C_{\beta} \sup _{v_{h}^{c} \neq 0} \frac{\left|\ell\left(v_{h}^{c}\right)-\hat{a}\left(\Pi_{h}^{C} u_{h}, v_{h}^{c}\right)\right|}{\left\|v_{h}^{c}\right\|_{1, \Omega}} .
$$

Since $u_{h}$ satisfies (2.6) for $v_{h}=v_{h}^{c}$ and $\left.\hat{a}_{h}\right|_{V_{h}^{c} \times V_{h}^{c}}=\left.\hat{a}\right|_{V_{h}^{c} \times V_{h}^{c}}$, it holds

$$
\ell\left(v_{h}^{c}\right)-\hat{a}\left(\Pi_{h}^{C} u_{h}, v_{h}^{c}\right)=\hat{a}_{h}\left(u_{h}-\Pi_{h}^{C} u_{h}, v_{h}^{c}\right) .
$$

Using (2.18) in (2.17) as well as (2.11b), we find

$$
\left\|u_{h}^{c}-\Pi_{h}^{C} u_{h}\right\|_{1, \Omega} \leq C_{\beta} C_{1}\left\|u_{h}-\Pi_{h}^{C} u_{h}\right\|_{1, h, \Omega}
$$


The assertion then follows from (2.16), (2.19), and (2.13).

We consider the residual-type a posteriori error estimator

$$
\eta_{h}:=\left(\sum_{T \in \mathcal{T}_{h}(\Omega)} \eta_{T}^{2}+\sum_{E \in \mathcal{E}_{h}\left(\Omega \cup \Gamma_{D}\right)} \eta_{E, 1}^{2}+\sum_{E \in \mathcal{E}_{h}\left(\Gamma_{R}\right)} \eta_{E, 2}^{2}\right)^{1 / 2},
$$

consisting of the element residuals

$$
\eta_{T}:=h_{T}\left\|f+\Delta u_{h}+k^{2} u_{h}\right\|_{0, T}, \quad T \in \mathcal{T}_{h}(\Omega),
$$

and the edge residuals

$$
\begin{aligned}
& \eta_{E, 1}:=h_{E}\left\|\left[\frac{\partial u_{h}}{\partial \nu_{E}}\right]_{E}\right\|_{0, E}, \quad E \in \mathcal{E}_{h}\left(\Omega \cup \Gamma_{D}\right), \\
& \eta_{E, 2}:=h_{E}\left\|g-\frac{\partial u_{h}}{\partial \nu_{E}}-i k u_{h}\right\|_{0, E}, \quad E \in \mathcal{E}_{h}\left(\Gamma_{R}\right) .
\end{aligned}
$$

As marking strategy for refinement we use Dörfler marking [16], i.e., given a constant $0<\theta<1$, we compute a set $\mathcal{M}_{1}$ of elements $T \in \mathcal{T}_{h}(\Omega)$ and a set $\mathcal{M}_{2}$ of edges $E \in \mathcal{E}_{h}(\bar{\Omega})$ such that

$$
\theta \eta_{h} \leq \tilde{\eta}_{h}:=\left(\sum_{T \in \mathcal{M}_{1}} \eta_{T}^{2}+\sum_{E \in \mathcal{M}_{2}}\left(\eta_{E, 1}^{2}+\eta_{E, 2}^{2}\right)\right)^{1 / 2} .
$$

Once the sets $\mathcal{M}_{i}, 1 \leq i \leq 2$, have been determined, a refined triangulation is generated based on newest vertex bisection [36].

3. Control of the consistency error, estimator reduction, and reliability. The following result shows that the upper bound for the consistency error can be controlled by the error estimator (cf. [9]). The proof follows the arguments of Lemma 3.6 in [9], but will be given for completeness.

Lemma 3.1. There exists a constant $C_{J}>0$, depending only on the shape regularity of $\mathcal{T}_{h}(\Omega)$, such that for $\alpha \geq \alpha_{2}:=2 C_{J} / \gamma$ it holds

$$
\alpha \eta_{h, c}^{2} \leq 2 \frac{C_{J}}{\gamma} \eta_{h}^{2}
$$

Proof. In view of (2.11a) and (2.6) with $v_{h}=u_{h}-\Pi_{h}^{C} u_{h}$, we obtain

$$
\begin{aligned}
\alpha \eta_{h, C}^{2} \leq & \left\|u_{h}-\Pi_{h}^{C} u_{h}\right\|_{1, h, \Omega}^{2} \leq \gamma^{-1} a_{h}^{I P}\left(u_{h}-\Pi_{h}^{C} u_{h}, u_{h}-\Pi_{h}^{C} u_{h}\right) \\
= & \gamma^{-1}\left(\sum_{T \in \mathcal{T}_{h}(\Omega)}\left(f+k^{2} u_{h}, u_{h}-\Pi_{h}^{C} u_{h}\right)_{0, T}\right. \\
& \left.+\sum_{E \in \mathcal{E}_{h}\left(\Gamma_{R}\right)}\left(g-i k u_{h}, u_{h}-\Pi_{h}^{C} u_{h}\right)_{0, E}-a_{h}^{I P}\left(\Pi_{h}^{C} u_{h}, u_{h}-\Pi_{h}^{C} u_{h}\right)\right) .
\end{aligned}
$$

Observing $L\left(\Pi_{h}^{C} u_{h}\right)=0,\left[\Pi_{h}^{C} u_{h}\right]_{E}=0$, for the last term on the right-hand side of 
(3.2) it follows that

$$
\begin{aligned}
& a_{h}^{I P}\left(\Pi_{h}^{C} u_{h}, u_{h}-\Pi_{h}^{C} u_{h}\right)=\sum_{T \in \mathcal{T}_{h}(\Omega)}\left(\nabla \Pi_{h}^{C} u_{h}, \nabla\left(u_{h}-\Pi_{h}^{C} u_{h}\right)\right)_{0, T} \\
& -\sum_{T \in \mathcal{T}_{h}(\Omega)} \overline{\left(L\left(u_{h}\right), \nabla \Pi_{h}^{C} u_{h}\right)_{0, T}}=\sum_{T \in \mathcal{T}_{h}(\Omega)}\left(\nabla u_{h}, \nabla\left(u_{h}-\Pi_{h}^{C} u_{h}\right)\right)_{0, T} \\
& -\sum_{T \in \mathcal{T}_{h}(\Omega)}\left\|\nabla\left(u_{h}-\Pi_{h}^{C} u_{h}\right)\right\|_{0, T}^{2}-\sum_{T \in \mathcal{T}_{h}(\Omega)} \overline{\left(L\left(u_{h}\right), \nabla\left(\Pi_{h}^{C} u_{h}\right)\right)_{0, T} .}
\end{aligned}
$$

An elementwise application of Green's formula reveals

$$
\begin{aligned}
& a_{h}^{I P}\left(\Pi_{h}^{C} u_{h}, u_{h}-\Pi_{h}^{C} u_{h}\right)=\sum_{T \in \mathcal{T}_{h}(\Omega)}\left(-\Delta u_{h}, u_{h}-\Pi_{h}^{C} u_{h}\right)_{0, T} \\
& +\sum_{E \in \mathcal{E}_{h}\left(\Omega \cup \Gamma_{D}\right)}\left(\nu_{E} \cdot\left[\nabla u_{h}\right]_{E},\left\{u_{h}-\Pi_{h}^{C} u_{h}\right\}_{E}\right)_{0, E}+\sum_{E \in \mathcal{E}_{h}\left(\Gamma_{R}\right)}\left(g-i k u_{h}, u_{h}-\Pi_{h}^{C} u_{h}\right)_{0, E} \\
& -\sum_{T \in \mathcal{T}_{h}(\Omega)}\left\|\nabla\left(u_{h}-\Pi_{h}^{C} u_{h}\right)\right\|_{0, T}^{2}+\sum_{T \in \mathcal{T}_{h}(\Omega)} \frac{\left(L\left(u_{h}\right), \nabla\left(u_{h}-\Pi_{h}^{C} u_{h}\right)\right)_{0, T}}{(L)}
\end{aligned}
$$

Using (3.3) and (3.4) in (3.2), straightforward estimation yields

$$
\begin{aligned}
& a_{h}^{I P}\left(u_{h}-\Pi_{h}^{C} u_{h}, u_{h}-\Pi_{h}^{C} u_{h}\right) \lesssim \eta_{h}\left(\left(\sum_{T \in \mathcal{T}_{h}(\Omega)} h_{T}^{-1}\left\|u_{h}-\Pi_{h}^{C} u_{h}\right\|_{0, T}^{2}\right)^{1 / 2}\right. \\
& \left.+\left(\sum_{E \in \mathcal{E}_{h}\left(\Omega \cup \Gamma_{D}\right)} h_{E}^{-1}\left\|u_{h}-\Pi_{h}^{C} u_{h}\right\|_{0, E}^{2}\right)^{1 / 2}\right)+\sum_{T \in \mathcal{T}_{h}(\Omega)}\left\|\nabla\left(u_{h}-\Pi_{h}^{C} u_{h}\right)\right\|_{0, T}^{2} \\
& +\left(\sum_{T \in \mathcal{T}_{h}(\Omega)}\left\|L\left(u_{h}\right)\right\|_{0, T}^{2}\right)^{1 / 2}\left(\sum_{T \in \mathcal{T}_{h}(\Omega)}\left\|\nabla\left(u_{h}-\Pi_{h}^{C} u_{h}\right)\right\|_{0, T}^{2}\right)^{1 / 2} .
\end{aligned}
$$

The stability (2.9) of the extension operator $L$ and the local approximation properties (2.13) of $\Pi_{h}^{C}$ imply the existence of $C_{J}>0$ such that

$$
\alpha \eta_{h, c}^{2} \leq \frac{C_{J}}{\gamma}\left(\eta_{h}^{2}+\eta_{h, c}^{2}\right)
$$

which readily leads to the assertion. $\mathbf{D}$

As a by-product of the preceding lemma we obtain the following results:

Corollary 3.2. Let $u_{h} \in V_{h}$ be the IPDG solution of (2.6), let $u_{h}^{c} \in V_{h}^{c}$ be the solution of (2.7), and let $u_{h}^{n c}:=u_{h}-u_{h}^{c}$. Then, there exists a constant $C_{c e}>0$, depending on $\gamma, C_{\gamma}$, and $C_{J}$, such that

$$
\left\|u_{h}^{n c}\right\|_{1, h, \Omega}^{2} \leq \frac{C_{c e}}{\alpha} \eta_{h}^{2}
$$

Proof. With $C_{c e}:=\left(2\left(1+C_{n c} C_{J}\right) / \gamma\right.$ the assertion is an immediate consequence of Lemma 2.1 and Lemma 3.1.

Corollary 3.3. Let $\mathcal{T}_{h}(\Omega)$ be a simplicial triangulation obtained by refinement from $\mathcal{T}_{H}(\Omega)$, and let $u_{h} \in V_{h}, u_{H} \in V_{H}$ and $\eta_{h}, \eta_{H}$ be the associated IPDG solutions 
of (2.6) and error estimators, respectively. Moreover, let $u_{h}^{c} \in V_{h}^{c}$ and $u_{H}^{c} \in V_{H}^{c}$ be the conforming approximations of (1.1a)-(1.1c) according to (2.7). Then, for $u_{h}^{n c}:=$ $u_{h}-u_{h}^{c}$ and $u_{H}^{n c}:=u_{H}-u_{H}^{c}$ we have

$$
\left\|u_{h}^{n c}-u_{H}^{n c}\right\|_{1, h, \Omega}^{2} \leq 4 \frac{C_{c e}}{\alpha}\left(\eta_{h}^{2}+\eta_{H}^{2}\right) .
$$

Proof. The triangle inequality yields

$$
\left\|u_{h}^{n c}-u_{H}^{n c}\right\|_{1, h, \Omega}^{2} \leq 2\left(\left\|u_{h}^{n c}\right\|_{1, h, \Omega}^{2}+\left\|u_{H}^{n c}\right\|_{1, h, \Omega}^{2}\right) .
$$

Taking

$$
\sum_{E \in \mathcal{E}_{h}} \frac{1}{h_{E}}\left\|\left[u_{H}^{n c}\right]_{E}\right\|_{0, E}^{2} \leq 2 \sum_{E \in \mathcal{E}_{H}} \frac{1}{H_{E}}\left\|\left[u_{H}^{n c}\right]_{E}\right\|_{0, E}^{2}
$$

into account and using Corollary 3.2 with $h$ replaced by $H$, we find

$$
\left\|u_{H}^{n c}\right\|_{1, h, \Omega}^{2} \leq 2 \frac{C_{c e}}{\alpha} \eta_{H}^{2} .
$$

We conclude by using (3.8) and (3.10) in (3.9).

The residual estimator $\eta_{h}$ has the following monotonicity property

$$
\eta_{h} \leq \eta_{H}
$$

for all refinements $\mathcal{T}_{h}(\Omega)$ of $\mathcal{T}_{H}(\Omega)$. The latter can be used to prove the following estimator reduction result which will be strongly used for the contraction property in section 5 .

LEMma 3.4. Let $\mathcal{T}_{h}(\Omega)$ be a simplicial triangulation obtained by refinement from $\mathcal{T}_{H}(\Omega)$, and let $u_{h} \in V_{h}, u_{H} \in V_{H}$, and $\eta_{h}, \eta_{H}, \tilde{\eta}_{H}$ be the associated IPDG solutions and error estimators, respectively. Then, for any $\tau>0$ there exists a constant $C_{\tau}>0$, depending only on the shape regularity of the triangulations, such that

$$
\eta_{h}^{2} \leq(1+\tau)\left(\eta_{H}^{2}-\left(1-2^{-1 / 2}\right) \tilde{\eta}_{H}^{2}\right)+C_{\tau} \sum_{T \in \mathcal{T}_{h}(\Omega)}\left\|\nabla\left(u_{h}-u_{H}\right)\right\|_{0, T}^{2} .
$$

Proof. The proof can be done along the same lines as the proof of Corollary 3.4 in $[11]$.

COROLlaRY 3.5. Under the same assumptions as in Lemma 3.4 let $\tau(\theta):=$ $(1+\tau)\left(1-2^{-1 / 2}\right) \theta$ with $\theta$ from $(2.23)$. Then, it holds

$$
\eta_{h}^{2} \leq \tau(\theta) \eta_{H}^{2}+C_{\tau} \sum_{T \in \mathcal{T}_{h}(\Omega)}\left\|\nabla\left(u_{h}-u_{H}\right)\right\|_{0, T}^{2}
$$

Proof. The proof is a direct consequence of (2.23) and (3.12).

Using, e.g., the unified approach to the a posteriori error control of IPDG methods [10], the reliability of the estimator $\eta_{h}$ can be easily established. 
Lemma 3.6. Let $u \in V$ and $u_{h} \in V_{h}$ be the solution of (2.2) and (2.6), respectively, and let $\xi$ and $\eta_{h}, \eta_{h, C}$ be the consistency error, the a posteriori error estimator, and the jump term as given by (2.12),(2.20), and (2.14). Then, there exists a constant $C_{r e l}>0$, depending only on the shape regularity of the triangulations, such that there holds

$$
a_{h}^{I P}\left(u-u_{h}, u-u_{h}\right) \leq C_{r e l} \eta_{h}^{2}
$$

Proof. The upper bound

$$
a_{h}^{I P}\left(u-u_{h}, u-u_{h}\right) \lesssim \eta_{h}^{2}+\xi^{2}
$$

can be derived as in [10]. Then, (3.14) follows readily from (2.14) and (3.1).

4. Quasi-orthogonality. Besides the reliability of the estimator and the estimator reduction result, a quasi-orthogonality property is a further important ingredient of the convergence analysis (cf. $[9,32,25]$ ). Here, the derivation of such a property is complicated due to the presence of the lower order term in the Helmholtz equation (1.1a). Adopting an idea from [22] (cf. also [39]) for the time-harmonic Maxwell equations, we resort to an Aubin-Nitsche type argument for the associated conforming approximation of the screen problem. As will be seen below, this additionally involves the error between the IPDG approximation and its conforming counterpart.

4.1. Mesh perturbation result. In the convergence analysis of IPDG methods for second order elliptic boundary value problems, mesh perturbation results estimating the coarse mesh error in the fine mesh energy norm from above by its coarse mesh energy norm have played a central role in the convergence analysis as a prerequisite for establishing a quasiorthogonality result (cf., e.g., [9, 25, 32]). Here, we provide the following mesh perturbation result:

LEMma 4.1. Let $\mathcal{T}_{h}(\Omega)$ be a simplicial triangulation obtained by refinement from $\mathcal{T}_{H}(\Omega)$. Then, for any $0<\varepsilon_{1}<1$ and $v \in V+V_{H}$ it holds

$$
a_{h}^{I P}(v, v) \leq\left(1+\varepsilon_{1}\right) a_{H}^{I P}(v, v)+\left(\frac{C_{L}}{\gamma \varepsilon_{1}}+1\right)\left(\eta_{h, C}^{2}+\eta_{H, C}^{2}\right) .
$$

Proof. For $v \in V+V_{H}$ we have

$$
\begin{aligned}
& a_{h}^{I P}(v, v)=\sum_{T \in \mathcal{T}_{h}(\Omega)}\|\nabla v\|_{0, T}^{2}+\sum_{E \in \mathcal{E}_{h}\left(\Omega \cup \Gamma_{D}\right)} \frac{\alpha}{h_{E}}\left\|[v]_{E}\right\|_{0, E}^{2} \\
& -2 \sum_{T \in \mathcal{T}_{h}(\Omega}\left((\operatorname{Re}(L(v)), \operatorname{Re}(\nabla v))_{0, T}+\left(\operatorname{Im}(L(v)), \operatorname{Im}(\nabla v)_{0, T}\right) .\right.
\end{aligned}
$$

Obviously, the following relationships hold true

$$
\begin{aligned}
\sum_{T \in \mathcal{T}_{h}(\Omega)}|v|_{1, T}^{2} & =\sum_{T \in \mathcal{T}_{H}(\Omega)}|v|_{1, T}^{2}, \\
\sum_{E \in \mathcal{E}_{h}\left(\Omega \cup \Gamma_{D}\right)} \frac{\alpha}{h_{E}}\left\|[v]_{E}\right\|_{0, E, h}^{2} & \leq 2 \sum_{E \in \mathcal{E}_{H}\left(\Omega \cup \Gamma_{D}\right)} \frac{\alpha}{H_{E}}\left\|[v]_{E}\right\|_{0, E, H}^{2} .
\end{aligned}
$$


Using (4.3a) in (4.2), we find

$$
\begin{aligned}
& a_{h}^{I P}(v, v)=a_{H}^{I P}(v, v)+\sum_{E \in \mathcal{E}_{h}\left(\Omega \cup \Gamma_{D}\right)} \frac{\alpha}{h_{E}}\left\|[v]_{E}\right\|_{0, E}^{2}-\sum_{E \in \mathcal{E}_{H}\left(\Omega \cup \Gamma_{D}\right)} \frac{\alpha}{H_{E}}\left\|[v]_{E}\right\|_{0, E}^{2} \\
& -2 \sum_{T \in \mathcal{T}_{h}(\Omega)}\left((\operatorname{Re}(L(v)), \operatorname{Re}(\nabla v))_{0, T}+(\operatorname{Im}(L(v)), \operatorname{Im}(\nabla v))_{0, T}\right) \\
& +2 \sum_{T \in \mathcal{T}_{H}(\Omega)}\left((\operatorname{Re}(L(v)), \operatorname{Re}(\nabla v))_{0, T}+(\operatorname{Im}(L(v)), \operatorname{Im}(\nabla v))_{0, T}\right) .
\end{aligned}
$$

The assertion follows by using Young's inequality in (4.4) and taking (2.9),(2.11a), and $(4.3 \mathrm{a}),(4.3 \mathrm{~b})$ into account.

4.2. Lower order term. The following result, which will be strongly needed in the derivation of the quasi-orthogonality result (cf. Theorem 4.1 below), is concerned with an estimate of the lower order term

$$
2 k^{2} \operatorname{Re}\left(c\left(u-u_{h}^{c}, u_{h}^{c}-u_{H}^{c}\right)+i k r\left(u-u_{h}^{c}, u_{h}^{c}-u_{H}^{c}\right)\right)
$$

where $u_{h}^{c} \in V_{h}^{c}, u_{H}^{c} \in V_{H}^{c}$ are the conforming approximations of (2.2). The proof uses the following regularity assumption:

(A) The solution $u$ of $(2.2)$ is $(1+r)$-regular for some $r \in(1 / 2,1]$, i.e., it satisfies $u \in V \cap H^{1+r}(\Omega)$ and for some positive constant $C$ it holds

$$
\|u\|_{1+r, \Omega} \leq C\left(\|f\|_{0, \Omega}+\|g\|_{0, \Gamma_{R}}\right) .
$$

LEMma 4.2. Let $\mathcal{T}_{h}(\Omega)$ be a simplicial triangulation obtained by refinement from $\mathcal{T}_{H}(\Omega)$ and let $u_{h}^{c} \in V_{h}^{c}, u_{H}^{c} \in V_{H}^{c}$ be the conforming approximations of (2.2). Then, under assumption $(\mathbf{A})$, there exists a constant $C_{L T}>0$, depending on the local geometry of the triangulations, such that

$$
\begin{aligned}
& 2 \operatorname{Re}\left(k^{2} c\left(u-u_{h}^{c}, u_{h}^{c}-u_{H}^{c}\right)+i k r\left(u-u_{h}^{c}, u_{h}^{c}-u_{H}^{c}\right)\right) \leq \\
& C_{L T} h^{r}\left(\left|u-u_{h}^{c}\right|_{1, \Omega}^{2}+\left|u_{h}^{c}-u_{H}^{c}\right|_{1, \Omega}^{2}\right) .
\end{aligned}
$$

Proof. Using a trace inequality, by straightforward estimation we deduce the existence of a constant $C_{L 1}>0$ such that

$$
\begin{aligned}
& 2 k^{2} \operatorname{Re}\left(c\left(u-u_{h}^{c}, u_{h}^{c}-u_{H}^{c}\right)+i k r\left(u-u_{h}^{c}, u_{h}^{c}-u_{H}^{c}\right)\right) \\
& \leq C_{L 1}\left|u-u_{h}^{c}\right|_{1, \Omega}\left(\left\|u_{h}^{c}-u_{H}^{c}\right\|_{0, \Omega}+\left\|u_{h}^{c}-u_{H}^{c}\right\|_{0, \Gamma_{R}}\right) .
\end{aligned}
$$

We define $z^{c} \in V$ as the solution of

$$
\begin{aligned}
& a\left(v^{c}, z^{c}\right)-k^{2} c\left(v^{c}, z^{c}\right)+i k r\left(v^{c}, z^{c}\right) \\
& =\left(u_{h}^{c}-u_{H}^{c}, v^{c}\right)_{0, \Omega}+\left(u_{h}^{c}-u_{H}^{c}, v^{c}\right)_{0, \Gamma_{R}}, \quad v^{c} \in V .
\end{aligned}
$$

Due to the regularity result (4.5), we have $z^{c} \in V \cap H^{1+r}(\Omega)$ and there exists a constant $C_{R}>0$ depending on the domain $\Omega$ such that

$$
\left\|z^{c}\right\|_{1+r, \Omega} \leq C_{R}\left(\left\|u_{h}^{c}-u_{H}^{c}\right\|_{0, \Omega}+\left\|u_{h}^{c}-u_{H}^{c}\right\|_{0, \Gamma_{R}}\right) .
$$


Choosing $v^{c}=u_{h}^{c}-u_{H}^{c}$ in (4.8) and observing Galerkin orthogonality, the trace inequality, the interpolation estimate

$$
\left\|z^{c}-I_{h} z^{c}\right\|_{1, \Omega} \leq C_{I} h^{r}\left\|z^{c}\right\|_{1+r, \Omega}
$$

and (4.10), we deduce the existence of a constant $C_{L 2}>0$, depending on $C_{I}, C_{R}$, and $C_{T}$ such that

$$
\begin{aligned}
& 2^{-1}\left(\left\|u_{h}^{c}-u_{H}^{c}\right\|_{0, \Omega}+\left\|u_{h}^{c}-u_{H}^{c}\right\|_{0, \Gamma_{R}}\right)^{2} \leq\left\|u_{h}^{c}-u_{H}^{c}\right\|_{0, \Omega}^{2}+\left\|u_{h}^{c}-u_{H}^{c}\right\|_{0, \Gamma_{R}}^{2}= \\
& a\left(u_{h}^{c}-u_{H}^{c}, z^{c}\right)-k^{2} c\left(u_{h}^{c}-u_{H}^{c}, z^{c}\right)+i k r\left(u_{h}^{c}-u_{H}^{c}, z^{c}\right)= \\
& a\left(u_{h}^{c}-u_{H}^{c}, z^{c}-I_{h} z^{c}\right)-k^{2} c\left(u_{h}^{c}-u_{H}^{c}, z^{c}-I_{h} z^{c}\right)+i k r\left(u_{h}^{c}-u_{H}^{c}, z^{c}-I_{h} z^{c}\right) \\
& \leq C_{L 2} h^{r}\left|u_{h}^{c}-u_{H}^{c}\right|_{1, \Omega}\left(\left\|u_{h}^{c}-u_{H}^{c}\right\|_{0, \Omega}+\left\|u_{h}^{c}-u_{H}^{c}\right\|_{0, \Gamma_{R}}\right),
\end{aligned}
$$

whence

$$
\left\|u_{h}^{c}-u_{H}^{c}\right\|_{0, \Omega}+\left\|u_{h}^{c}-u_{H}^{c}\right\|_{0, \Gamma_{R}} \leq 2 C_{L 2} h^{r}\left|u_{h}^{c}-u_{H}^{c}\right|_{1, \Omega} .
$$

Hence, choosing $C_{L T}:=4 C_{L 1} C_{L 2}$, the assertion follows from (4.7) and (4.11).

4.3. Quasiorthogonality. In this subsection, we prove the following quasiorthogonality result:

THEOREM 4.1. Let $\mathcal{T}_{h}(\Omega)$ be a simplicial triangulation obtained by refinement from $\mathcal{T}_{H}(\Omega)$, and let $u_{h} \in V_{h}, u_{H} \in V_{H}$ and $\eta_{h}, \eta_{H}$ be the associated solutions of (2.6) and error estimators, respectively. Further, let $e_{h}:=u-u_{h}$ and $e_{H}:=u-u_{H}$ be the fine and coarse mesh errors. Then, for any $0<\varepsilon<1$ there exists a meshwidth $h_{\max }>0$, depending on the wavenumber $k$, the domain $\Omega$ and $\varepsilon$, and a constant $C_{Q}>0$, depending on $\gamma, C_{1}, C_{c e}, C_{L T}$, and $k$, such that for all $h \leq h_{\max }$ it holds

$$
\begin{aligned}
& a_{h}^{I P}\left(e_{h}, e_{h}\right) \leq \\
& (1+\varepsilon) a_{H}^{I P}\left(e_{H}, e_{H}\right)-\frac{\gamma}{8}\left\|u_{h}-u_{H}\right\|_{1, h, \Omega}^{2}+\frac{C_{Q}}{\alpha}\left(\eta_{h}^{2}+\eta_{H}^{2}\right) .
\end{aligned}
$$

Proof. With $u_{h}^{c} \in S_{h}$ and $u_{H}^{c} \in S_{H}$ as the conforming P1 approximations of (2.2) with respect to the triangulations $\mathcal{T}_{h}(\Omega)$ and $\mathcal{T}_{H}(\Omega)$ we have

$$
\begin{aligned}
& a_{h}^{I P}\left(e_{h}, e_{h}\right)=a_{h}^{I P}\left(e_{h}+u_{h}^{c}-u_{H}^{c}, e_{h}+u_{h}^{c}-u_{H}^{c}\right) \\
& -2 \operatorname{Re} a_{h}^{I P}\left(e_{h}, u_{h}^{c}-u_{H}^{c}\right)-a_{h}^{I P}\left(u_{h}^{c}-u_{H}^{c}, u_{h}^{c}-u_{H}^{c}\right) .
\end{aligned}
$$

The three terms on the right-hand side in (4.13) will be estimated separately. These estimates will be provided by the following three lemmas.

Lemma 4.3. Under the same assumptions as in Theorem 4.1 there exists a constant $C_{2}>0$, depending on $\gamma, C_{1}, C_{c e}, C_{J}$, and $C_{L}$, such that for any $0<\hat{\varepsilon}<1 / 2$ there holds

$$
a_{h}^{I P}\left(e_{h}+u_{h}^{c}-u_{H}^{c}, e_{h}+u_{h}^{c}-u_{H}^{c}\right) \leq(1+\hat{\varepsilon}) a_{H}^{I P}\left(e_{H}, e_{H}\right)+\frac{C_{2}}{\alpha}\left(\eta_{h}^{2}+\eta_{H}^{2}\right) .
$$


Proof. We split the first term on the right-hand side of (4.13) according to

$$
\begin{aligned}
& a_{h}^{I P}\left(u-u_{h}+u_{h}^{c}-u_{H}^{c}, u-u_{h}+u_{h}^{c}-u_{H}^{c}\right)= \\
& a_{h}^{I P}\left(e_{H}+u_{H}^{n c}-u_{h}^{n c}, e_{H}+u_{H}^{n c}-u_{h}^{n c}\right) .
\end{aligned}
$$

Using (2.11b), Young's inequality, and Corollary 3.3, we find

$$
\begin{aligned}
& a_{h}^{I P}\left(e_{H}+u_{H}^{n c}-u_{h}^{n c}, e_{H}+u_{H}^{n c}-u_{h}^{n c}\right) \\
& \leq a_{h}^{I P}\left(e_{H}, e_{H}\right)+C_{1}\left\|u_{h}^{n c}-u_{H}^{n c}\right\|_{1, h, \Omega}^{2}+2 C_{1}^{1 / 2} a_{h}^{I P}\left(e_{H}, e_{H}\right)^{1 / 2}\left\|u_{h}^{n c}-u_{H}^{n c}\right\|_{1, h, \Omega} \\
& \leq\left(1+\varepsilon_{2}\right) a_{h}^{I P}\left(e_{H}, e_{H}\right)+C_{1}\left(1+\frac{1}{\varepsilon_{2}}\right)\left\|u_{h}^{n c}-u_{H}^{n c}\right\|_{1, h, \Omega}^{2} \\
& \leq\left(1+\varepsilon_{2}\right) a_{h}^{I P}\left(e_{H}, e_{H}\right)+4 C_{1} \frac{C_{c e}}{\alpha}\left(1+\frac{1}{\varepsilon_{2}}\right)\left(\eta_{h}^{2}+\eta_{H}^{2}\right) .
\end{aligned}
$$

For the first term on the right-hand side in (4.16), the mesh perturbation result (4.1) and a subsequent application of (2.14) tell us

$$
a_{h}^{I P}\left(e_{H}, e_{H}\right) \leq\left(1+\varepsilon_{1}\right) a_{H}^{I P}\left(e_{H}, e_{H}\right)+\frac{2 C_{J} C_{L}}{\alpha \varepsilon_{1} \gamma^{2}}\left(\eta_{h}^{2}+\eta_{H}^{2}\right) .
$$

Choosing $0<\varepsilon_{i}<1,1 \leq i \leq 2$, such that $\hat{\varepsilon}:=\varepsilon_{1}+\varepsilon_{2}+\varepsilon_{1} \varepsilon_{2}<1 / 2$, and

$$
C_{2}:=2\left(1+\varepsilon_{2}\right) \frac{C_{J} C_{L}}{\varepsilon_{1} \gamma^{2}}+4 C_{1} C_{c e}\left(1+\frac{1}{\varepsilon_{2}}\right),
$$

the assertion follows from (4.16) and (4.17).

Lemma 4.4. Under the same assumptions as in Theorem 4.1, there exists a constant $C_{i}>0,3 \leq i \leq 6$, depending on $\gamma, C_{c e}$, and $C_{L T}$, such that

$$
\begin{aligned}
& 2 \operatorname{Re} a_{h}^{I P}\left(e_{h}, u_{h}^{c}-u_{H}^{c}\right) \leq \\
& C_{3} h^{r} a_{h}^{I P}\left(e_{h}, e_{h}\right)+\left(\frac{\gamma}{4}+C_{4} h^{r}\right)\left\|u_{h}-u_{H}\right\|_{1, h, \Omega}^{2}+\frac{C_{5}+C_{6} h^{r}}{\alpha}\left(\eta_{h}^{2}+\eta_{H}^{2}\right),
\end{aligned}
$$

where $C_{3}:=2 C_{L T} / \gamma, C_{4}:=3 C_{L T}$, and the positive constants $C_{5}, C_{6}$ depend on the wavenumber $k$ and on $C_{c e}, C_{L T}$.

Proof. For the second term on the right-hand side of (4.13) we have

$$
\begin{aligned}
& \left.2 \operatorname{Re}\left(a_{h}^{I P}\left(e_{h}, u_{h}^{c}-u_{H}^{c}\right)\right)=2 \operatorname{Re}\left(k^{2} c\left(e_{h}, u_{h}^{c}-u_{H}^{c}\right)+i k r_{h}\left(e_{h}, u_{h}^{c}-u_{H}^{c}\right)\right)\right)= \\
& 2 \operatorname{Re}\left(\left(k^{2} c\left(u-u_{h}^{c}, u_{h}^{c}-u_{H}^{c}\right)+i k r\left(u-u_{h}^{c}, u_{h}^{c}-u_{H}^{c}\right)\right)\right)+ \\
& \left.2 \operatorname{Re}\left(k^{2} c\left(u_{h}^{c}-u_{h}, u_{h}^{c}-u_{H}^{c}\right)+i k r\left(u_{h}^{c}-u_{h}, u_{h}^{c}-u_{H}^{c}\right)\right)\right) .
\end{aligned}
$$

In view of Lemma 4.2, the first term on the right-hand side in (4.19) can be estimated as follows

$$
\begin{aligned}
& 2 \operatorname{Re}\left(\left(k^{2} c\left(u-u_{h}^{c}, u_{h}^{c}-u_{H}^{c}\right)+i k r\left(u-u_{h}^{c}, u_{h}^{c}-u_{H}^{c}\right)\right)\right. \\
& \leq C_{L T} h^{r}\left(\left|e_{h}^{c}\right|_{1, h, \Omega}^{2}+\left|u_{h}^{c}-u_{H}^{c}\right|_{1, h, \Omega}^{2}\right) .
\end{aligned}
$$


Taking advantage of (2.11a) and Corollary 3.3, for the two terms on the right-hand side in (4.20) we find

$$
\begin{aligned}
\left|e_{h}^{c}\right|_{1, h, \Omega}^{2} & \leq 2\left(\left\|u-u_{h}\right\|_{1, h, \Omega}^{2}+\left\|u_{h}-u_{h}^{c}\right\|_{1, h, \Omega}^{2}\right) \\
& \leq \frac{2}{\gamma} a_{h}^{I P}\left(e_{h}, e_{h}\right)+2 \frac{C_{c e}}{\alpha} \eta_{h}^{2}, \\
\left|u_{h}^{c}-u_{H}^{c}\right|_{1, h, \Omega}^{2} & \leq 3\left(\left\|u_{h}-u_{H}\right\|_{1, h, \Omega}^{2}+\left\|u_{h}-u_{h}^{c}\right\|_{1, h, \Omega}^{2}+\left\|u_{H}-u_{H}^{c}\right\|_{1, h, \Omega}^{2}\right) \\
& \leq 3\left\|u_{h}-u_{H}\right\|_{1, h, \Omega}^{2}+6 \frac{C_{c e}}{\alpha}\left(\eta_{h}^{2}+\eta_{H}^{2}\right) .
\end{aligned}
$$

and hence,

$$
\begin{aligned}
& 2 \operatorname{Re}\left(k^{2} c\left(u-u_{h}^{c}, u_{h}^{c}-u_{H}^{c}\right)+i k r\left(u-u_{h}^{c}, u_{h}^{c}-u_{H}^{c}\right)\right) \\
& \leq \frac{2}{\gamma} C_{L T} h^{r} a_{h}^{I P}\left(e_{h}, e_{h}\right)+3 C_{L T} h^{r}\left\|u_{h}-u_{H}\right\|_{1, h, \Omega}^{2}+8 \frac{C_{c e} C_{L T}}{\alpha} h^{r}\left(\eta_{h}^{2}+\eta_{H}^{2}\right) .
\end{aligned}
$$

We split the second term on the right-hand side in (4.19) according to

$$
\begin{aligned}
2 \operatorname{Re} & \left(k^{2} c_{h}\left(u_{h}^{c}-u_{h}, u_{h}^{c}-u_{H}^{c}\right)+i k r_{h}\left(u_{h}^{c}-u_{h}, u_{h}^{c}-u_{H}^{c}\right)\right) \\
= & 2 \operatorname{Re}\left(k^{2} c_{h}\left(u_{h}^{c}-u_{h}, u_{h}^{c}-u_{h}\right)+i k r_{h}\left(u_{h}^{c}-u_{h}, u_{h}^{c}-u_{h}\right)\right) \\
& +2 \operatorname{Re}\left(k^{2} c_{h}\left(u_{h}^{c}-u_{h}, u_{h}-u_{H}\right)+i k r_{h}\left(u_{h}^{c}-u_{h}, u_{h}-u_{H}\right)\right) \\
& +2 \operatorname{Re}\left(k^{2} c_{h}\left(u_{h}^{c}-u_{h}, u_{H}-u_{H}^{c}\right)+i k r_{h}\left(u_{h}^{c}-u_{h}, u_{H}-u_{H}^{c}\right)\right) .
\end{aligned}
$$

By (3.7) and Young's inequality, the three terms on the right-hand side in (4.22) can be estimated as follows

$$
\begin{aligned}
& 2 \operatorname{Re}\left(k^{2} c_{h}\left(u_{h}^{c}-u_{h}, u_{h}^{c}-u_{h}\right)+i k r_{h}\left(u_{h}^{c}-u_{h}, u_{h}^{c}-u_{h}\right)\right) \\
& \leq 4 \max \left(k, k^{2}\right) \frac{C_{c e}}{\alpha} \eta_{h}^{2}, \\
& 2 \operatorname{Re}\left(k^{2} c_{h}\left(u_{h}^{c}-u_{h}, u_{h}-u_{H}\right)+i k r_{h}\left(u_{h}^{c}-u_{h}, u_{h}-u_{H}\right)\right) \\
& \leq \frac{\gamma}{4}\left\|u_{h}-u_{H}\right\|_{1, h, \Omega}^{2}+\frac{4 C_{c e}}{\alpha \gamma}\left(\max \left(k, k^{2}\right)\right)^{2} \eta_{h}^{2}, \\
& 2 \operatorname{Re}\left(k^{2} c_{h}\left(u_{h}^{c}-u_{h}, u_{H}-u_{H}^{c}\right)+i k r_{h}\left(u_{h}^{c}-u_{h}, u_{H}-u_{H}^{c}\right)\right) \\
& \leq 2 \frac{C_{c e}}{\alpha} \max \left(k, k^{2}\right)\left(\eta_{h}^{2}+\eta_{H}^{2}\right) .
\end{aligned}
$$

Then, (4.18) follows from (4.19)-(4.22) and the preceding estimates.

Lemma 4.5. Under the same assumptions as in Theorem 4.1, there exists a constant $C_{7}>0$ such that

$$
a_{h}^{I P}\left(u_{h}^{c}-u_{H}^{c}, u_{h}^{c}-u_{H}^{c}\right) \geq \frac{\gamma}{2}\left\|u_{h}-u_{H}\right\|_{1, h, \Omega}^{2}-\frac{C_{7}}{\alpha}\left(\eta_{h}^{2}+\eta_{H}^{2}\right) .
$$


Proof. Taking into account (2.11a) and using Young's inequality and (3.7) we find

$$
\begin{aligned}
& a_{h}^{I P}\left(u_{h}^{c}-u_{H}^{c}, u_{h}^{c}-u_{H}^{c}\right) \geq \gamma\left\|u_{h}^{c}-u_{H}^{c}\right\|_{1, h, \Omega}^{2} \geq \gamma\left(\left\|u_{h}-u_{H}\right\|_{1, h, \Omega}^{2}+\right. \\
& \left.\left\|u_{h}^{c}-u_{h}+u_{H}-u_{H}^{c}\right\|_{1, h, \Omega}^{2}-2\left|\left(u_{h}-u_{H}, u_{h}^{c}-u_{h}+u_{H}-u_{H}^{c}\right)_{1, h, \Omega}\right|\right) \\
& \geq\left(\gamma-\frac{\varepsilon}{2}\right)\left\|u_{h}-u_{H}\right\|_{1, h, \Omega}^{2}-4 \gamma \varepsilon^{-1}\left(\left\|u_{h}^{n c}\right\|_{1, h, \Omega}^{2}+\left\|u_{H}^{n c}\right\|_{1, h, \Omega}^{2}\right) \\
& \geq\left(\gamma-\frac{\varepsilon}{2}\right)\left\|u_{h}-u_{H}\right\|_{1, h, \Omega}^{2}-4 \gamma \frac{C_{c e}}{\alpha \varepsilon}\left(\eta_{h}^{2}+\eta_{H}^{2}\right) .
\end{aligned}
$$

Then, (4.23) follows from (4.24) for $\varepsilon=\gamma$ with $C_{7}:=4 C_{c e}$. $\square$

Proof of Theorem 4.1. Using the estimates from Lemma 4.3, Lemma 4.4, and 4.5 in (4.13), we obtain

$$
\begin{aligned}
a_{h}^{I P}\left(e_{h}, e_{h}\right) \leq & \frac{1+\hat{\varepsilon}}{1-C_{3} h^{r}} a_{H}^{I P}\left(e_{H}, e_{H}\right)-\frac{\gamma / 4-c_{4} h^{r}}{1-C_{3} h^{r}}\left\|u_{h}-u_{H}\right\|_{1, h, \Omega}^{2} \\
& +\frac{C_{5}+C_{6} h^{r}+C_{7}}{\alpha\left(1-C_{3} h^{r}\right)}\left(\eta_{h}^{2}+\eta_{H}^{2}\right) .
\end{aligned}
$$

We choose $h_{\max }>0$ such that

$$
\frac{1+\hat{\varepsilon}}{1-C_{3} h_{\max }^{r}} \leq 1+2 \hat{\varepsilon}, \quad \frac{\gamma / 4-c_{4} h_{\max }^{r}}{1-C_{3} h_{\max }^{r}} \geq \gamma / 8
$$

Then, (4.12) follows from (4.25) with $\varepsilon:=2 \hat{\varepsilon}$ and $C_{Q}:=\left(C_{5}+C_{6} h_{\max }^{r}+C_{7}\right) /(1-$ $C_{3} h_{\text {max }}^{r}$.

5. Contraction property. We now use the monotonicity result (3.12) and the quasiorthogonality (4.12) to prove the following contraction property:

TheOREM 5.1. Let $u \in H_{0, \Gamma_{D}}^{1}(\Omega)$ be the unique solution of (2.2). Further, let $\mathcal{T}_{h}(\Omega)$ be a simplicial triangulation obtained by refinement from $\mathcal{T}_{H}(\Omega)$, and let $u_{h} \in$ $V_{h}, u_{H} \in V_{H}$ and $\eta_{h}, \eta_{H}$ be the associated solutions of (2.6) and error estimators, respectively. Then, there exist constants $0<\delta<1$ and $\rho>0$, depending only on the shape regularity of the triangulations and the parameter $\theta$ from the Dörfler marking, such that for sufficiently large penalty parameter $\alpha$ and sufficiently small mesh widths $h, H$ the fine mesh and coarse mesh discretization errors $e_{h}:=u-u_{h}$ and $e_{H}=u-u_{H}$ satisfy

$$
a_{h}^{I P}\left(e_{h}, e_{h}\right)+\rho \eta_{h}^{2} \leq \delta\left(a_{H}^{I P}\left(e_{H}, e_{H}\right)+\rho \eta_{H}^{2}\right) .
$$

Proof. Multiplying the estimator reduction property (3.13) by $\gamma /\left(8 C_{\tau}\right)$ and substituting the result into the quasi-orthogonality estimate (4.12), for $\rho>0$ we get

$$
\begin{aligned}
a_{h}^{I P}\left(e_{h}, e_{h}\right)+\rho \eta_{h}^{2} \leq & (1+\varepsilon) a_{H}^{I P}\left(e_{H}, e_{H}\right) \\
& +\left(\frac{C_{Q}}{\alpha}-\frac{\gamma}{8 C_{\tau}}+\rho\right) \eta_{h}^{2}+\left(\frac{C_{Q}}{\alpha}+\frac{\gamma \tau(\theta)}{8 C_{\tau}}\right) \eta_{H}^{2} .
\end{aligned}
$$

For the choice

$$
\alpha>\frac{8 C_{Q} C_{\tau}}{\gamma}, \quad \rho:=\frac{\gamma}{8 C_{\tau}}-\frac{C_{Q}}{\alpha}
$$


it follows from (5.2) that

$$
a_{h}^{I P}\left(e_{h}, e_{h}\right)+\rho \eta_{h}^{2} \leq(1+\varepsilon) a_{H}^{I P}\left(e_{H}, e_{H}\right)+\left(\frac{C_{Q}}{\alpha}+\frac{\gamma \tau(\theta)}{8 C_{\tau}}\right) \eta_{H}^{2} .
$$

Invoking the reliability (3.14) of the estimator, we find

$$
\begin{aligned}
& a_{h}^{I P}\left(e_{h}, e_{h}\right)+\rho \eta_{h}^{2} \leq \\
& \left.\delta a_{H}^{I P}\left(e_{H}, e_{H}\right)+((1+\varepsilon)-\delta) a_{H}^{I P}\left(e_{H}, e_{H}\right)+\frac{\gamma \tau(\theta)}{8 C_{\tau}}\right) \eta_{H}^{2} \leq \\
& \delta a_{H}^{I P}\left(e_{H}, e_{H}\right)+\left(C_{r e l}((1+\varepsilon)-\delta)+\frac{C_{Q}}{\alpha}+\frac{\gamma \tau(\theta)}{8 C_{\tau}}\right) \eta_{H}^{2} .
\end{aligned}
$$

We choose $\delta$ such that

$$
\rho=\frac{\gamma}{8 C_{\tau}}-\frac{C_{Q}}{\alpha}=\delta^{-1}\left(C_{r e l}((1+\varepsilon)-\delta)+\frac{C_{Q}}{\alpha}+\frac{\gamma \tau(\theta)}{8 C_{\tau}}\right) .
$$

Solving for $\delta$, we obtain

$$
\delta=\frac{C_{r e l}(1+\varepsilon)+\frac{C_{Q}}{\alpha}+\frac{\gamma \tau(\theta)}{8 C_{\tau}}}{\frac{\gamma}{8 C_{\tau}}-\frac{C_{Q}}{\alpha}+C_{r e l}} .
$$

Now, we choose

$$
\begin{aligned}
\tau & =\tau^{*}:=\frac{1}{2} \frac{\left(1-2^{-1 / 2}\right) \theta}{1-\left(1-2^{-1 / 2}\right) \theta}<\frac{1}{4} \\
\varepsilon & :=\frac{1}{2} \frac{\gamma\left(1-\tau^{*}\right)}{8 C_{r e l} C_{\tau^{*}}}<1 .
\end{aligned}
$$

It follows that

$$
\delta=\frac{C_{r e l}+\frac{\gamma\left(1+\tau^{*}\right)}{16 C_{\tau^{*}}}+\frac{C_{Q}}{\alpha}}{C_{r e l}+\frac{\gamma}{8 C_{\tau^{*}}}-\frac{C_{Q}}{\alpha}}
$$

Looking for $\alpha$ such that

$$
\frac{\gamma\left(1+\tau^{*}\right)}{16 C_{\tau^{*}}}+\frac{C_{Q}}{\alpha}<\frac{\gamma}{8 C_{\tau *}}-\frac{C_{Q}}{\alpha},
$$

we find that $0<\delta<1$ for

$$
\alpha>\frac{32 C_{Q} C_{\tau^{*}}}{\left(1-\tau^{*}\right) \gamma}
$$

This concludes the proof of the contraction property.

6. Numerical Results. We present a documentation of numerical results for two examples. In order to illustrate the convergence history of the adaptive IPDG approach in terms of the exact discretization error $e_{h}:=u-u_{h}$ in the mesh dependent energy norm $a_{h}^{I P}\left(e_{h}, e_{h}\right)^{1 / 2}$, as a first example we choose an interior Dirichlet problem for the Helmholtz equation where the exact solution is known. In particular, we consider (1.1a) in a bounded polygonal domain $\Omega \subset \mathbb{R}^{2}$ with the boundary conditions 
(1.1b),(1.1c) replaced by a Dirichlet boundary condition on $\Gamma:=\partial \Omega$. We note that the preceding convergence analysis applies to such interior Dirichlet problems as well.

Example 1: We choose $\Omega$ as the L-shaped domain $\Omega:=(-1,+1)^{2} \backslash[0,+1) \cup(-1,0]$ and consider the interior Dirichlet problem

$$
\begin{array}{rlrl}
-\Delta u-k^{2} u & =f & & \text { in } \Omega, \\
u=g & & \text { on } \Gamma .
\end{array}
$$

The source terms $f, g$ are chosen such that $u(r, \varphi)=J_{1 / 2}(k r)$ (in polar coordinates) is the exact solution, where $J_{1 / 2}$ stands for the Bessel function of the first kind. The solution is an oscillating function with decreasing amplitude for increasing $r$ which exhibits a singularity at the origin (cf. Fig. 6.1 (left)).
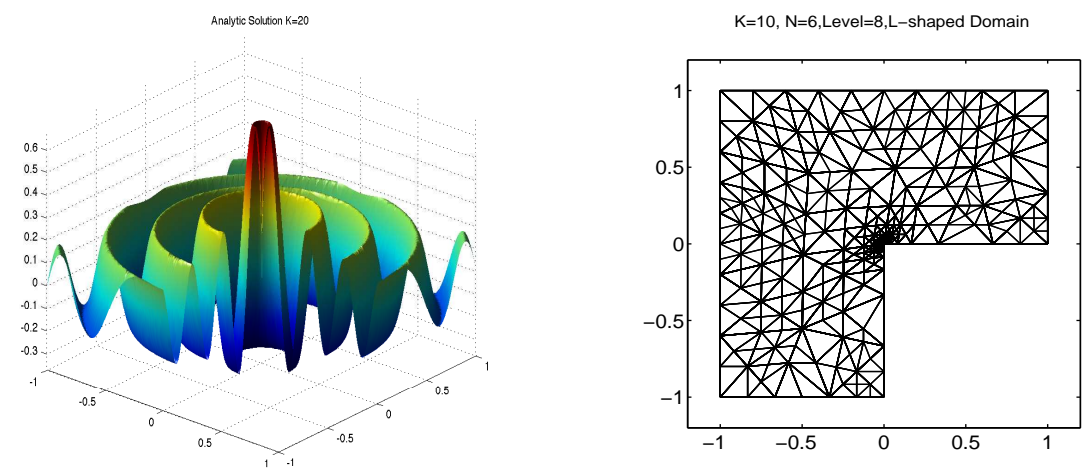

FIG. 6.1. Exact solution for $k=20$ (left) and adaptively refined grid after 8 refinement steps for $k=10, N=6$, and $\theta=0.3$ (right).
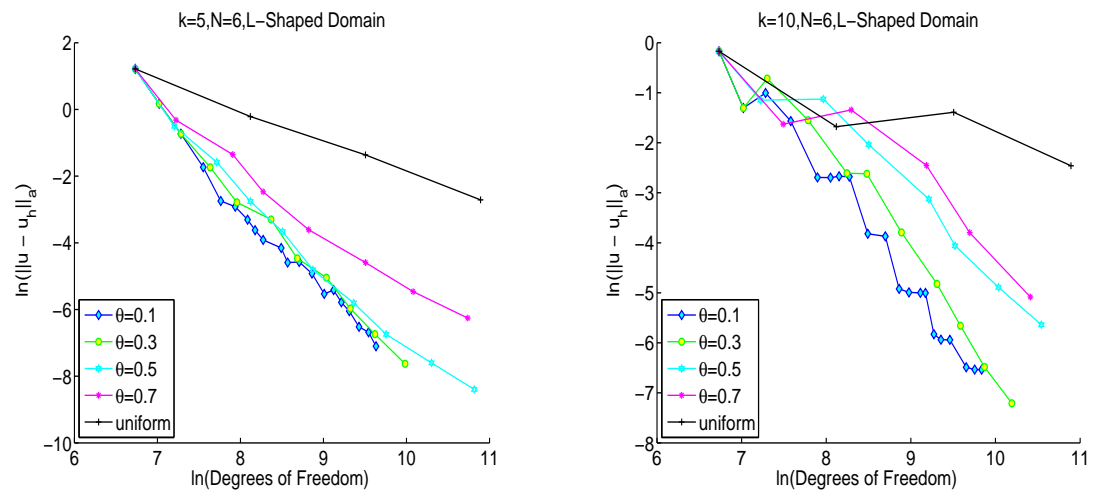

FIG. 6.2. Convergence history of the adaptive IPDG method. Mesh dependent energy error as a function of the DOF (degrees of freedom) on a logarithmic scale: $k=5, N=6$ (left) and $k=10$, $N=6$ (left).

We have applied the adaptive IPDG method to (6.1a),(6.1b). For $k=10, N=6$, and $\theta=0.3$, Figure 6.1 (right) shows the adaptively refined mesh after 8 refinement 
steps with a pronounced refinement in a vicinity of the singularity at the origin.

Figure 6.2 reflects the convergence history of the adaptive process. The mesh dependent energy norm $\left\|u-u_{h}\right\|_{a}:=a_{h}^{I P}\left(u-u_{h}, u-u_{h}\right)^{1 / 2}$ of the error is displayed as a function of the total number of degrees of freedom on a logarithmic scale. The curves represent the decrease in the error both for uniform refinement and for adaptive refinement in case of different values of the constant $\theta$ in the Dörfler marking. In particular, Figure 6.2 (left) refers to the wavenumber $k=5$ and the polynomial degree $N=6$, whereas Figure 6.2 (right) shows the results for the wavenumber $k=10$ and the same polynomial degree $N=6$. As for adaptive IPDG applied to standard second order elliptic boundary value problems (cf. [25]) we observe optimal convergence rates for small $\theta$. Moreover, as can be expected, for a high wavenumber the asymptotic regime is reached later, i.e., for finer meshes, compared to lower wavenumbers.

The second example deals with the screen problem (1.1a)-(1.1c).

Example 2: We choose $\Omega:=(-1,+1)^{2} \backslash\left(S_{1} \cup S_{2}\right)$ where

$$
\begin{aligned}
& S_{1}:=\operatorname{conv}((0,0),(-0.25,+0.50),(-0.50,+0.50)), \\
& S_{2}:=\operatorname{conv}((0,0),(+0.25,-0.50),(+0.50,-0.50)),
\end{aligned}
$$

such that $\Gamma_{R}=\partial(-1,+1)^{2}$ and $\Gamma_{D}:=\partial S_{1} \cup \partial S_{2}$. The right-hand sides $f$ and $g$ are chosen according to $f \equiv 0$ and

$$
g=\cos \left(k x_{2}\right)+i \sin \left(k x_{2}\right) .
$$

The real part of the computed IPDG approximation is shown in Figure 6.3 for wavenumber $k=15$ (left) and for wavenumber $k=20$ (right).
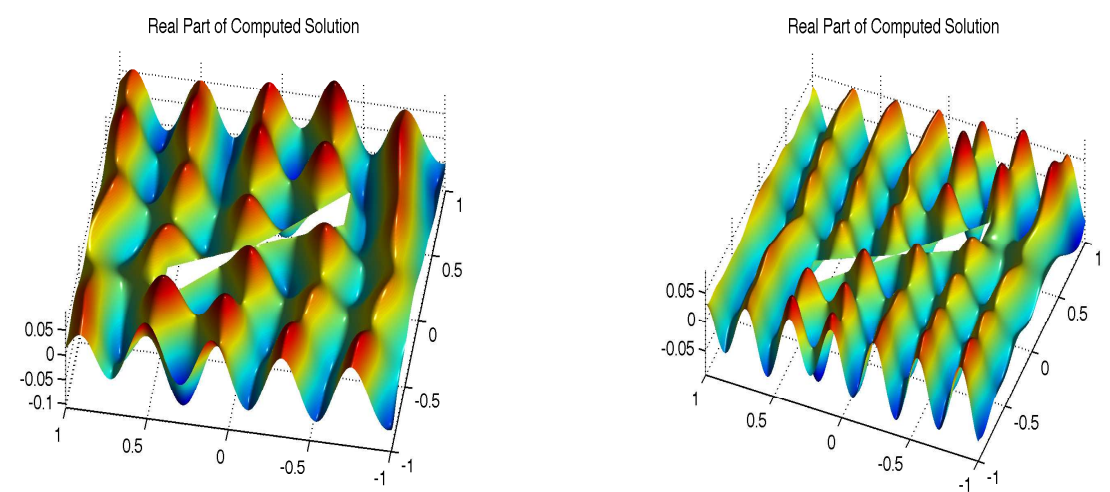

FIG. 6.3. Real part of the computed IPDG approximation for $k=15$ (left) and $k=20$ (right).

Figure 6.4 contains the adaptively refined mesh for wavenumber $k=10$ and polynomial degree $N=6$ after 12 refinement steps (left) and for wavenumber $k=20$ and polynomial degree $N=6$ after 8 refinement steps (right).

Since we do not have access to the exact solution of the screen problem, we document the convergence history of the adaptive IPDG method by representing the decrease in the error estimator $\eta_{h}$ as a function of the total number of degrees of freedom on a logarithmic scale. In particular, Figure 6.5 shows the results for wavenumber $k=10$ and polynomial degree $N=4$ (left) resp. polynomial degree 
$\mathrm{N}=6$ Level 12,Screen Problem

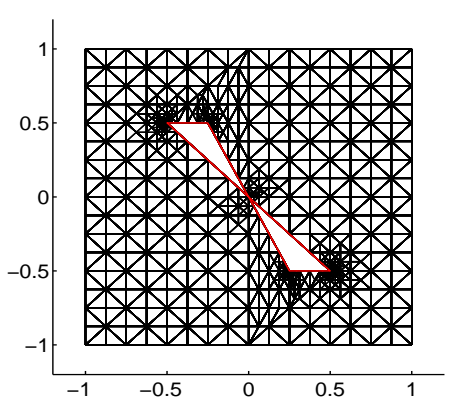

$\mathrm{N}=6$ Level 8,Screen Problem

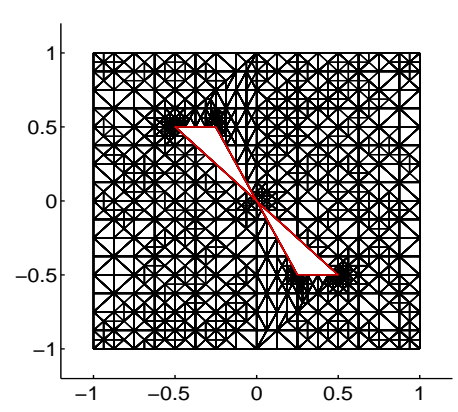

FIG. 6.4. Adaptively refined mesh for $k=10, N=6$ after 8 refinement steps (left) and for $k=20, N=6$ after 12 refinement steps (right).
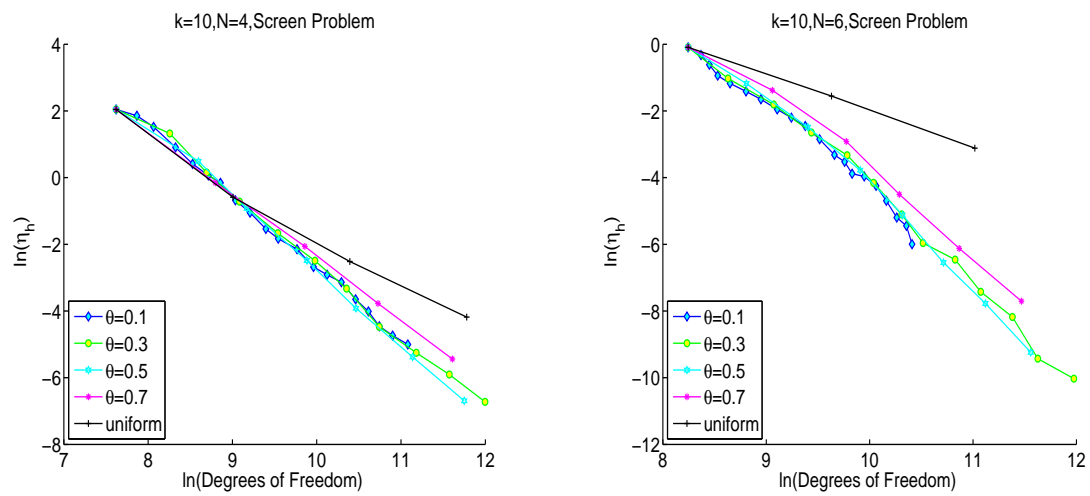

FIG. 6.5. Convergence history of the adaptive IPDG method. Error estimator as a function of the DOF (degrees of freedom) on a logarithmic scale: $k=10, N=4$ (left) and $k=10, N=6$ (left).

$N=6$ (right). Likewise, Figure 6.6 displays the convergence history for wavenumber $k=15$ and polynomial degrees $N=4$ (left) and $N=6$ (right). We observe a similar behavior as in case of the interior Dirichlet problem (Example 1). For higher wavenumbers, the asymptotic regimes require fines meshes. Moreover, as we expect, higher polynomial degrees can handle higher wave numbers better at the expense of increased computational work.

\section{REFERENCES}

[1] M. Ainsworth, A posteriori error estimation for Discontinuous Galerkin finite element approximation. SIAM J. Numer. Anal. 45, 1777-1798, 2007.

[2] M. Amara, R. Djellouli, and C. Farhat, Convergence analysis of a discontinuous Galerkin method with plane waves and lagrange multipliers for the solution of Helmholtz problems, SIAM J. Numer. Anal. 47, 10381066, 2009.

[3] G. B. Alvarez, A. F. D. Loula, E. G. Dutra do Carmo, and F. A. Rochinha, A discontinuous finite element formulation for Helmholtz equation. Comput. Methods Appl. Mech. Engrg. 

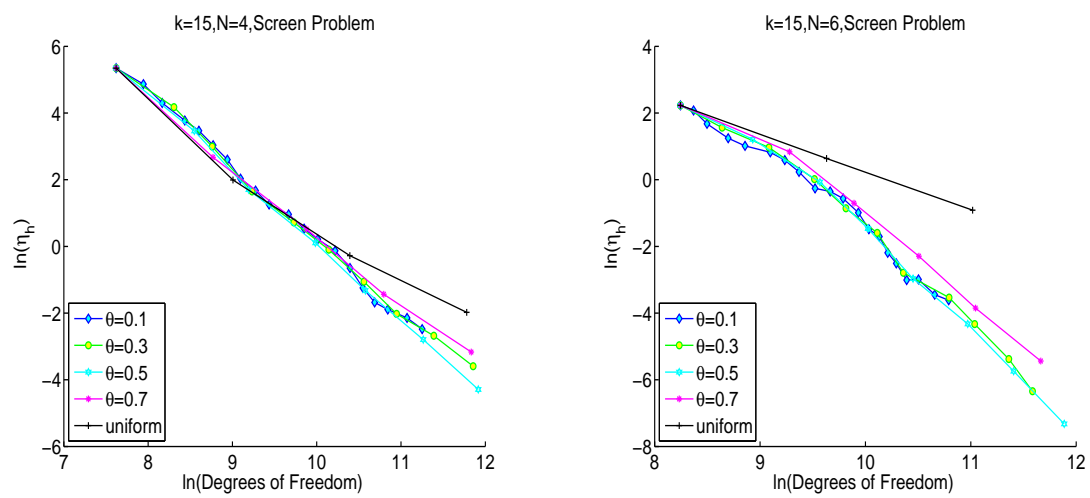

FIG. 6.6. Convergence history of the adaptive IPDG method. Error estimator as a function of the DOF (degrees of freedom) on a logarithmic scale: $k=15, N=4$ (left) and $k=15, N=6$ (left).

195, 4018-4035, 2006.

[4] A. K. Aziz and A. Werschulz, On the numerical solutions of Helmholtzs equation by the finite element method. SIAM J. Numer. Anal. 17, 681-686, 1980.

[5] I. Babuska and S. Sauter, Is the pollution effect of the FEM avoidable for the Helmholtz equation?. SIAM Review 42, (2000), 451-484, 2000.

[6] I. Babuska and T. Strouboulis, The Finite Element Method and its Reliability. Clarendon Press, Oxford, 2001.

[7] W. Bangerth and R. Rannacher, Adaptive Finite Element Methods for Differential Equations. Lectures in Mathematics. ETH-Zürich. Birkhäuser, Basel, 2003.

[8] R. Becker, P. Hansbo, and M.G. Larson, Energy norm a posteriori error estimation for discontinuous Galerkin methods. Comput. Methods Appl. Mech. Engrg. 192, 723-733, 2003.

[9] A. Bonito and R. Nochetto, Quasi-optimal convergence rate of an adaptive Discontinuous Galerkin method. SIAM J. Numer. Anal. 48, 734-771, 2010.

[10] C. Carstensen, T. Gudi, and M. Jensen, A unifying theory of a posteriori error control for discontinuous Galerkin FEM. Numer. Math. 112, 363-379, 2009.

[11] J.M. Cascon, Ch. Kreuzer, R.H. Nochetto, and K.G. Siebert, Quasi-optimal rate of convergence of adaptive finite element methods. SIAM J. Numer. Anal. 46, 2524-2550, 2008.

[12] C. L. Chang, A least-squares finite element method for the Helmholtz equation. Comput. Methods Appl. Mech. Engrg. 83, 1-7, 1990.

[13] E. T. Chung and B. Engquist, Optimal discontinuous Galerkin methods for wave propagation. SIAM J. Numer. Anal. 44, 2131-2158, 2006.

[14] B. Cockburn, Discontinuous Galerkin methods. Z. Angew. Math. Mech. 83, 731-754, 2003.

[15] D. Colton and P. Monk, The numerical solution of the three-dimensional inverse scattering problem for time harmonic acoustic waves. SIAM J. Sci. Statist. Comput. 8, 278-291, 1987.

[16] Dörfler, W.; A convergent adaptive algorithm for Poisson's equation. SIAM J. Numer. Anal. 33, 1106-1124, 1996.

[17] B. Engquist and O. Runborg, Computational high frequency wave propagation. Acta Numer. 12, 181-266, 2003.

[18] X. Feng and H. Wu, Discontinuous Galerkin methods for the Helmholtz equation with large wave numbers. SIAM J. Numer. Anal. 47, 2872-2896, 2009.

[19] X. Feng and H. Wu, hp-discontinuous Galerkin methods for the Helmholtz equation with large wave number. submitted, arXiv:0907.3442v1, 2009.

[20] G. Gabard, Discontinuous Galerkin methods with plane waves for time-harmonic problems. J. Comp. Phys. 225, 19611984, 2007.

[21] C. Gittelson, R. Hiptmair, and I. Perugia, Plane wave discontinuous Galerkin methods: Analysis of the h-version. ESAIM: M2AN Math. Model. Numer. Anal. 43, 297-331, 2009.

[22] J. Gopalakrishnan and J. Pasciak, Overlapping Schwarz preconditioners for indefinite time harmonic Maxwell equations. Math. Comp. 72, 1-15, 2003. 
[23] R. Griesmaier and P. Monk, Error analysis for a hybridizable discontinuous Galerkin method for the Helmholtz equation. J. Sci. Comp. (in press), 2011.

[24] J.S. Hesthaven and T. Warburton, Nodal Discontinuous Galerkin Methods. Springer, BerlinHeidelberg-New York, 2008.

[25] R.H.W. Hoppe, G. Kanschat, and T. Warburton, Convergence analysis of an adaptive interior penalty discontinuous Galerkin method. SIAM J. Numer. Anal., 47, 534-550, 2009.

[26] P. Houston, D. Schötzau, and T.P. Wihler, Energy norm a posteriori error estimation of hpadaptive discontinuous Galerkin methods for elliptic problems. Math. Models Methods Appl. Sci. 17, 33-62, 2007.

[27] F. Ihlenburg, Finite Element Analysis Of Acoustic Scattering. Springer, Berlin-Heidelberg-New York, 1998.

[28] F. Ihlenburg and I. Babuska, Finite element solution of the Helmholtz equation with high wave number. II. The h-p version of the FEM. SIAM J. Numer. Anal. 34, 315-358, 1997.

[29] N. A. Kampanis, J. Ekaterinaris, and V. Dougalis, Effective Computational Methods for Wave Propagation. Chapman \& Hall/CRC, Boca Raton, 2008.

[30] P. Monk and D.Wang, A least squares method for the Helmholtz equation, Computer Methods in Applied Mechanics and Engineering 175. 121-136, 1999.

[31] O. Karakashian and F. Pascal, A posteriori error estimates for a discontinuous Galerkin approximation of second-order elliptic problems. SIAM J. Numer. Anal. 41, 2374-2399, 2003.

[32] O. Karakashian and F. Pascal, Convergence of adaptive Discontinuous Galerkin approximations of second-order elliptic problems. SIAM J. Numer. Anal. 45, 641-665, 2007.

[33] J.-C. Nédélec, Acoustic and Electromagnetic Equations. Integral Representations for Harmonic Problems, Springer, Berlin-Heidelberg-New York, 2001.

[34] B. Rivière, Discontinuous Galerkin Methods for Solving Elliptic and Parabolic Equations. Theory and Implementation. SIAM, Philadelphia, 2008.

[35] B. Rivière and M.F. Wheeler, A posteriori error estimates and mesh adaptation strategy for discontinuous Galerkin methods applied to diffusion problems. Computers \& Mathematics with Applications 46, 141-163, 2003.

[36] A. Schmidt and K. G. Siebert, Design of Adaptive Finite Element Software: The Finite Element Toolboox ALBERTA. Springer, Berlin-Heidelberg-New York, 2005.

[37] D. Schötzau, C. Schwab, and A. Toselli, Mixed hp-dgfem for incompressible flows. SIAM J. Numer. Anal., 40, 2171-2194, 2003.

[38] L. Tartar, Introduction to Sobolev Spaces and Interpolation Theory. Springer, BerlinHeidelberg-New York, 2007.

[39] L. Zhong, L. Chen, S. Shu, G. Wittum, and J. Xu, Convergence and optimality of adaptive edge finite element methods for time-harmonic Maxwell equations. Preprint, Department of Mathematics, University of California at Irvine, 2010. 